\title{
Un même environnement, deux interprétations écopoétiques (chez les Peuls du Mali)
}

\section{Christiane Seydou}

\section{(2) OpenEdition}

1 Journals

Édition électronique

URL : https://journals.openedition.org/clo/8565

DOI : $10.4000 /$ clo.8565

ISSN : 2266-1816

Éditeur

INALCO

\section{Édition imprimée}

Date de publication : 30 juin 2020

Pagination : 147-174

ISBN : 978-2-85831-392-1

ISSN : 0396-891X

Référence électronique

Christiane Seydou, «Un même environnement, deux interprétations écopoétiques (chez les Peuls du Mali) », Cahiers de littérature orale [En ligne], 87 | 2020, mis en ligne le 16 septembre 2021, consulté le 09 novembre 2022. URL : http://journals.openedition.org/clo/8565; DOl : https://doi.org/10.4000/clo. 8565

\section{(c) (9) \&}

Creative Commons - Attribution - Pas d'Utilisation Commerciale 4.0 International - CC BY-NC 4.0

https://creativecommons.org/licenses/by-nc/4.0/ 


\section{Un même environnement, deux interprétations écopoétiques (chez les Peuls du Mali)}

Christiane SEYdou

Directeure de recherche honoraire au CNRS, Llacan, UMR 8135

S'il est évident que toute pensée, tout discours, toute réalisation ne sont jamais que les fruits d'un faisceau d'influences pérennes et de situations circonstancielles, il est tout aussi évident que la façon dont joue cette confluence signe les orientations fondatrices émanant de leur auteur, et que les modalités choisies pour les exprimer signifient le sens de la présence au monde de celui-ci, en en reflétant la situation socioculturelle et les traits identitaires.

Là réside le cœur de toute la problématique soulevée par une approche écopoétique de la production littéraire d'une communauté. L'exploration des rapports entre environnement et cette expression langagière à vocation esthétique et culturelle qu'est la littérature ouvre sur un carrefour de voies qui sont toutes aussi intéressantes et prodigues en éclaircissements sur le phénomène de la création littéraire.

Ainsi, peut-on explorer le traitement de l'environnement naturel dans les œuvres de tel ou tel auteur ou celui du grand thème de la nature au cours des siècles et dans divers pays; ou encore, en amont, comment tel environnement particulier a conditionné les sentiments, l'attitude d'une population dans ses options non seulement littéraires mais existentielles, etc.

À cela s'ajoute un paramètre supplémentaire qui s'avère fondamental : celui des différences de situations concernant les sociétés de culture écrite et celles de culture orale; chez ces dernières la création littéraire s'inscrit dans un système socioculturel global qui confère à l'œuvre une dimension pragmatique et une fonction collective qui en influencent la forme et la performance. Voilà 
ce qui explique l'importance du rapport entre l'environnement total et la création littéraire là où domine l'oralité, lieu de la communication et voie de la transmission qui assurent la cohésion et la perpétuation d'une communauté et de sa culture, inscrites, tant l'une que l'autre, dans un même milieu naturel prégnant.

On ne peut, dans le cas des « littératures orales » - oxymore désormais familier - se défaire de la complexité des interférences entre «l'environnement total » et le « texte émis », du fait que cet environnement intervient non seulement en tant que référent naturel et culturel constitutif du vécu et évoqué comme tel, dans le temps de la composition muette du texte, mais aussi en tant que décor réel, tout entier présent, lors de la profération du texte et - selon les circonstances et les destinataires - donnant sens à celui-ci, au-delà de sa simple fonction sémantique immédiate. Quant à l'expression formelle du « texte » ainsi inscrit dans cet entrelacs de réalités environnementales, elle obéit à des lois stylistiques - autrement dit à une esthétique - plus ou moins commandées par les exigences du contexte de la performance.

Dès lors, la perspective écopoétique intègre, ici, tout un réseau de niveaux qui interfèrent dans :

. les raisons contextuelles (environnement naturel, mais aussi socioculturel) qui motivent et conditionnent la conception du texte ;

- la place de cet environnement dans le texte et ses types de représentation ;

- les choix stylistiques impliqués par l'inscription du texte dans les conditions environnementales de son énonciation.

C'est ce qu'illustrent de façon particulièrement claire deux genres poétiques pratiqués par les Peuls du Massina, dans la Boucle du Niger ${ }^{1}$.

Cette région du Mali, souvent désignée comme le wuddu pulaaku, le « nombril de la foulanité ${ }^{2} »$, présente la particularité d'être une région où s'illustre clairement l'évidence de la coalescence totale entre nature et culture. De par sa réalité géographique, cette immense plaine est totalement soumise aux lois de la nature dans toutes ses composantes : d'une platitude à perte de vue et soumise au rythme des conditions climatiques, elle est envahie chaque année par la crue du Niger sur des centaines de kilomètres, et n'est ponctuée que de

\section{Cf. Seydou, 1991.}

2. Terme formé sur la racine ful-présente dans Pullo (pl. Fulbe). Au Mali, ce terme traduit selon le contexte « la gent peule » ou « l'identité, la culture, la manière d'être spécifique du Peul ». 
rares buttes exondées où se réfugient quelques villages ${ }^{3}$. Voilà ce qui contraint toute sa population, à une mobilité régulée par les rythmes saisonniers, mais aussi ce qui a conditionné toute la géographie ethnique du pays, chaque population s'inscrivant dans cet espace et dans ce temps en fonction de ses activités distinctives - agriculture, pêche, élevage - et, du même coup, dans un réseau de relations fécond tant dans la vie économique que dans les échanges sociaux, culturels et linguistiques ${ }^{4}$.

Nonobstant cette situation, le Massina a été, dans sa réalité historique, au cœur de l'un des quelques grands états centralisés de l'Afrique précoloniale : l'Empire peul du Massina ${ }^{5}$ ou Dîna et, de ce fait, a connu, outre l'établissement d'un système étatique théocratique, une organisation de l'espace exceptionnellement rigoureuse, marquée par une distribution spatiotemporelle planifiée des activités liées à la nature. C'est ainsi que la terre, soumise au flux et au reflux de la crue, est - outre zone bénie pour les pêcheurs - tour à tour lieu de culture de riz pour les agriculteurs et riche pâturage pour les éleveurs. Les Peuls, particulièrement impliqués dans la constitution et la gestion de cet «Empire » y ont gardé une position bivalente : bien que sédentarisés et participant à l'administration de la région, nombreux y sont aussi ceux qui pratiquent le pastoralisme. Et cette bivalence trouve un reflet dans deux genres littéraires pratiqués dans cette région ${ }^{6}$; l'étude de la production poétique chez sédentaires d'une part, et pasteurs de l'autre, s'avère fort éclairante, concernant les rapports entre environnement et littérature.

\section{Le genre mergol}

Un premier genre, pratiqué par qui en a le goût et le talent, porte le nom de mergol, substantif d'un verbe (merde) signifiant « parler de chose et d'autre, à bâtons rompus »; et de fait, le poète mergoowo traite des sujets les plus variés en jouant sur les moyens prosodiques et stylistiques habituels de la poésie libre et sur un mode de déclamation monocorde mais rythmé, combinant accents d'intensité

3. Voir Gallais, 1967 et 1984.

4.C'est ainsi que l'un des poètes peuls les plus célèbres de cette région, le mergoowo Ndjîdo Kawdo Tamboura, était, comme son nom l'indique, bambara.

5. Voir Bâ \& Daget, 1984, et Sanankoua, 1990.

6. Sont aussi richement cultivées une poésie religieuse ou mystique à formes fixes (empruntées à la poésie arabe classique) et comptant plusieurs genres, et une poésie profane de forme libre, la seule dont il sera question ici. 
et unités de souffle. La diversité des thèmes abordés commande le style et le rythme adoptés : cela peut aller, de la narration dramatique d'un affrontement armé ancien ou celle, quelque peu narquoise, d'un incident administratif plus récent $^{7}$, à l'énumération des compétences et des outils d'un travailleur ou à celle de tout ce que peut faire le $\mathrm{feu}^{8}$; de la description par le menu de la situation actuelle de la région, à la célébration des mérites d'un notable particulier, en recensant les lieux et les biens qu'il administre ; de l'évocation de la vie pastorale suscitée par l'éloge d'un généreux propriétaire de troupeau, à un recensement de toponymes regroupés par leurs initiales (soixante-dix-neuf noms, commençant par Ka-, Ma-, Sa-, Si-, Ta-...) ; ailleurs encore, ce seront les images audacieuses d'un pamphlet grivois ${ }^{9}$ !... Et les sujets s'enchaînent, rebondissant parfois sur un mot, pour s'engager dans un autre, totalement différent.

L'environnement représenté dans cette poésie étant total : naturel, technique, social, politique..., ce genre littéraire apparaît comme un moyen, pour le poète, tout en exhibant son talent, de préserver et transmettre tout à la fois l'ensemble de la culture et la richesse de la langue, faisant de ses mergi (pluriel de mergol) une sorte de conservatoire vivant. C'est ainsi qu'un poème dédié à un Bozo commence par le situer dans l'espace naturel, en enfilant les noms des lieux où il exerce ses talents de pêcheur : bras d'eau, gués, rives, etc.; puis, passant au registre technique, sont scandés les vingt-huit noms d'instruments de pêche utilisés. Tel autre poème évoquant l'environnement villageois, énumère tout ce que l'on trouve sur un marché ${ }^{10}$; ailleurs encore, un poète, pour justifier son autolouange, exhibe son talent, dédié cette fois à l'environnement linguistique, en débitant un lexique bilingue - bambara-peul, reflet de la pratique linguistique de cette région - de cent-soixante-douze mots dont la succession s'ordonne en une cascade d'échos, en fonction de la qualité sonore de chaque couple ${ }^{11}$ !

Toutefois, la représentation de l'espace tient, dans ce genre poétique, une place prédominante et constante, non en tant qu'évocation romantique de paysages inspirant sentiment esthétique ou souvenir émouvant, mais bien comme indice de localisation d'une action ou d'une gestion des activités s'y déroulant, et comme condition nécessaire du vécu et des attitudes y afférant. Chaque nom

7.Seydou, 1991, p. 314-335.

8. Ibid., p. 338-345.

9. Ibid., p. 348-357.

10. Voir le premier extrait présenté en Annexe.

11. Voir Seydou, 1989. 
propre cité est régulièrement suivi de la localisation de la personne qu'il désigne et, le cas échéant, de l'étendue du fief sur lequel elle exerce son autorité ; les toponymes aussi y abondent, souvent remplacés par les devises ${ }^{12}$ les désignant ; car c'est une coutume peule d'attribuer aux lieux des qualificatifs qui remplacent leur nom, en faisant le plus souvent référence à la végétation, au monde animal, à l'hydrographie, voire, pour ce qui est des villes, à l'architecture... ; les devises complètes sont présentes surtout dans les récits épiques; dans les mergi, le plus souvent, l'amorce de la devise ou bien simplement un qualificatif y figurant, suffit à désigner la ville dont le nom réel est absent : ainsi la ville de Ténenkou se trouve désignée par Awre-Burgu, « Première-née du Bourgou » ou Furde-Kodda, «Poussiéreuse du Benjamin »...

Mais ce sont surtout l'occupation de l'espace et sa distribution qui font l'objet de développements importants ; par exemple, le célèbre mergoowo Njîdo Kawdo Tamboura a récité un long mergol de sept cent quarante vers ${ }^{13}$ dans lequel il enchaîne les thèmes les plus variés : vie d'un arbre, de sa graine à sa souche, liste des attributs de Dieu, who's who de la gent poète pratiquant le mergol, succession d'aphorismes, listes de mots parmi lesquels une dizaine de noms d'oiseaux, récits d'un incident qui eut lieu en 1946 !... après quoi, il consacre deux cent treize vers au recensement détaillé des lieux attribués à chaque troupeau transhumant selon la fraction peule à laquelle il appartient : lieu de regroupement et de stationnement sur les îles en attendant le signal d'entrée dans les pâturages du Bourgou (zone de pâturage aquatique laissé par la décrue); points de traversée des bras d'eau réservés à chaque groupe ; trajets de pénétration et organisation de la dynamique opérationnelle pour l'occupation du territoire par les troupeaux.

De même, dans un autre très long poème dédié aux Chefs de Bourgou chargés de l'administration de chaque portion de cette aire, ce poète établit une sorte de cadastre, en présentant, pour chaque chef évoqué, les lieux dessinant son fief et en donnant quelques détails topographiques, puis, là encore, quelques itinéraires dévolus aux transhumants. Toutes ces données d'ordre trivialement pragmatique, mais investies de la portée esthétique du discours poétique, acquièrent, outre une forme séduisante, un potentiel mnémotechnique ; ce qui favorise la stabilité et la diffusion d'un savoir, garant d'un vécu serein, respectueux d'une tradition socioculturelle imposée essentiellement par l'écologie, conditionnée ici par situation topographique et condition pastorale : toute la vie s'inscrit dans les

12. Voir Seydou, 2013.

13. Voir Seydou, 1991, p. 190-249. 
contours de l'immense plaine envahie par la crue du fleuve, puis, à la décrue, par les hordes de bovins revenant jouir des riches pâturages laissés par le retrait des eaux, cette réalité «écologique » exigeant une connaissance solide d'une organisation stricte du temps et de l'espace pour assurer une conduite concertée des différents troupeaux, mais aussi, bien plus largement, contribuer à une gestion équilibrée de toute la vie économique de cette plaine du delta intérieur du Niger, conjuguant, selon les saisons, ressources halieutiques, agricoles (riziculture) et pastorales.

Et cette connaissance des multiples données constitutives du vécu se trouve divulguée au gré des déplacements des poètes ; car la déclamation des mergi s'effectue en toute occasion festive où le récitant peut rencontrer un public, voire, lorsqu'il rend visite à une personne impliquée dans l'organisation sociale, l'administration ou simplement dans l'une ou l'autre des activités ordinaires : pêche, élevage, commerce... ; ainsi, outre son caractère de performance poétique, ce genre revêt une fonction informative, la répétition et la circulation de ces textes participant au maintien d'un savoir collectif nécessaire et à la diffusion de l'actualité de toute la région.

L'activité de ces poètes, inscrite dans la vie quotidienne, tant par les sujets abordés que par les circonstances et les occasions de leurs séances de déclamation, apparaît comme une entreprise de sauvegarde des connaissances et des pratiques, une sorte d'Académie vivante, pragmatique, assurant leur diffusion et leur transmission sous les aspects d'un « gai savoir », entretenu et sans cesse enrichi grâce à la motivation sous-jacente et féconde d'une concurrence conventionnelle tacite ou revendiquée ${ }^{14}$ - entre poètes.

\section{Le genre jammooje na'i}

Tout autre est le cas du genre poétique appelé jammooje na'i ou « éloges/ devises des bovins » qui, lui, est inscrit dans une situation écologique totale qui conditionne non seulement ses thèmes, mais sa composition et sa performance : il n'est pas l'apanage de poètes indépendants, libres de choisir leurs sujets d'inspiration et leur style, mais la production de jeunes bergers qui, dans la solitude d'une longue transhumance, composent sur un sujet commun attendu

14. Ce genre est en effet traditionnellement placé sous le signe de la rivalité ; tel poète ponctue son poème de... : « je méduse les poètes... je suis imbattable... Hé ! Moi, je jette les poètes dans d'aveugles ténèbres (textuellement : "j'ai rendu anyctalopes les poètes"), quoi qu'ils fassent... » 
et suivant une stylistique établie, un poème qu'ils déclameront lors des fêtes de transhumance tout en faisant défiler leurs bêtes devant les villageois attroupés; cette exhibition festive n'est pas dépourvue d'un certain esprit de compétition puisqu'un jury décerne un fanion au berger dont le troupeau apparaît le mieux entretenu et le plus discipliné ; et s'il n’est pas de prix explicitement décerné au meilleur poète, la célébrité acquise en particulier auprès des jeunes filles par le plus doué, lui est récompense suffisante !

Quelle est ici la place de l'écologie ? Elle est on ne peut plus évidente puisqu'on ne peut dissocier l'auteur de son environnement - topographique, pédologique, atmosphérique, animal, végétal - dont il est partie intégrante; seul avec son troupeau et dans un cadre uniquement naturel voire « sauvage » durant ces mois de transhumance, le seul trait d'union qu'il lui reste avec son monde socioculturel est sa langue, ce bien inaliénable, quand bien même resté tacite en ces conditions particulières ; et si son isolement $\mathrm{du}$ monde humain plonge le berger dans cette imprégnation globale du monde naturel, le travail stylistique qu'exige la composition de son poème lui conserve au moins un contact avec cet élément culturel de base qu'est la langue; et sa prise de parole hors communication, car sans destinataire immédiat, lorsqu'il clame son poème pour le mémoriser, l'aide à « culturaliser » explicitement son vécu muet et ensauvagé du moment.

Il s'agit, en fait, de mettre en mots cette situation ordinaire, reconduite chaque année dans les mêmes conditions, et commune à tous les bergers, bien que vécue solitairement et individuellement; si bien que ces poèmes disent tous la même chose : la marche inlassable des troupeaux dans un décor et un climat sans grande surprise ; inscrits dans l'écoulement répétitif du travail et des saisons, ils ne comportent aucun signe de narrativité, n'ont ni introduction ni conclusion et sont purement descriptifs et vocatifs ; une analyse du lexique montre qu'un quart en est réservé aux seuls termes désignant et qualifiant les bovins (couleur des robes, emplacement et forme des taches sur la robe, forme et orientation des cornes, aspect du toupillon); et un cinquième se rapporte à l'environnement naturel en ses multiples champs sémantiques : eau, espace (qualité de la terre, végétation, topographie et toponymie, orientation...), temps atmosphérique et cosmique (soleil, vent, chaleur, jour et nuit, saison); quelques représentants du monde animal sauvage apparaissent aussi ça et là : oiseaux, insectes, fauves, divers ongulés... ; enfin un assez grand nombre de toponymes ponctuent l'évocation de cette déambulation ou encore, se déroulant en une suite arbitraire, ils ne décrivent aucun itinéraire ni ne situent quelque événement, ne se prêtant alors qu'au pur jeu de sonorités que permet la liberté de toute mise en liste, affranchie de toute contrainte syntaxique. Toutefois, cet environnement si présent n'est jamais décrit pour lui-même, mais dans son association indéfectible au vécu du troupeau et de 
son berger et, de ce fait, n'est qu'un décor mouvant traversé au gré des saisons, et comme un des éléments d'un univers global où tout est mouvement et incessant renouvellement, au gré des déplacements et des conditions atmosphériques.

Quel intérêt, alors, pour ces jeunes bergers, de mettre en paroles ce vécu « habituel », ordinaire et répétitif ? Tous vivant et disant la même chose, où situer, pour chacun, la dimension « poétique »- au double sens du terme : esthétique et créatif - de sa composition personnelle et le moyen de prouver ainsi son talent ? Comment affirmer son emprise culturelle sur cet environnement en faisant autre chose que simplement le dire dans sa banale réalité ? Cela se situera au niveau non plus du contenu du texte lui-même - éminemment évident -, mais à celui d'une manipulation stylistique particulière dont on va voir que, en nous faisant glisser d'une écopoétique à une « échopoétique », elle va nous projeter dans un au-delà des mots.

En effet, toute visée « informative » étant ici - à la différence du genre mergol - totalement superflue, le sens immédiat des mots cède le pas à leur réalité phonique et aux effets esthétiques qu'elle permet, pour donner à la dimension sémantique de la langue une portée accrue ; c'est ainsi que la caractéristique dominante de ce genre poétique est un enfilement des mots selon une technique faisant appel principalement aux jeux d'échos facilités par l'exploitation de leur face sonore ; toutefois, au recours à toutes les figures stylistiques les plus courantes - anaphores, épiphores, parallélismes, chiasmes, etc. - s'ajoute la recherche d'un effet particulier produit par une cascade d'allitérations se succédant de façon continue grâce à un tuilage qui favorise le glissement d'une allitération dominante à la suivante de façon progressive ; en évitant tout passage abrupt d'un environnement sonore à un autre, cette technique a comme résultat de créer un enchaînement ininterrompu de mots qui, par leurs constituants acoustiques, peuvent, sans solution de continuité, s'engendrer les uns les autres indéfiniment ${ }^{15}$. C'est ainsi que le jeune Allâye Tégué, dans son poème de 1073 vers, enfile la musique des mots dont on voit le tuilage assurer l'harmonieuse suc(cession :

15. Voir Seydou, 1991, p. 26-29 et un exemple en Annexe 2. Voir aussi Peuls du Mali, poésie pastorale, poésie religieuse. Vidéo CNRS (en ligne). 
Christiane SEYDOU

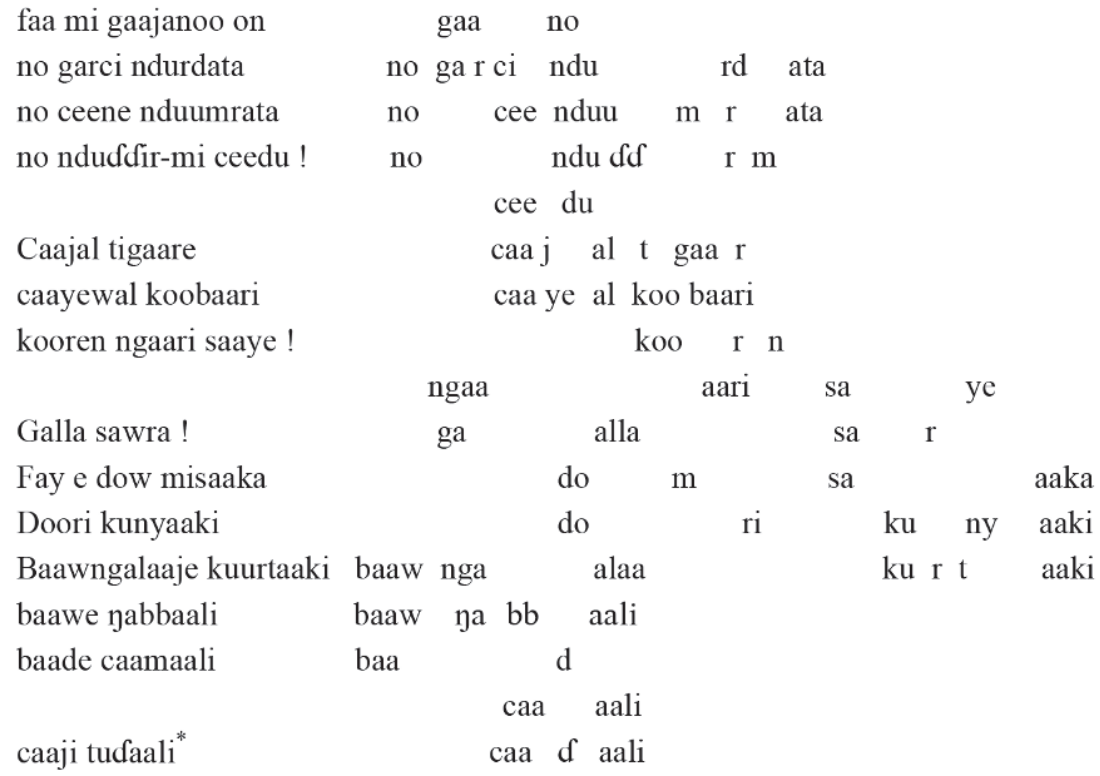

Cette utilisation experte de la langue comme un matériau esthétique sonore pour créer cette impression de continuité, associée à la façon dont le texte, en l'absence de toute narrativité, traduit à travers la monotonie du vécu, ce même continuum - vital pour l'économie pastorale -, trouve encore un écho concordant dans la situation et le style de profération de cette poésie.

Les circonstances de la performance de ce poème sont pourtant à l'inverse de celles de sa composition et de sa récitation solitaire pour sa mémorisation. Et pour son auteur, le renouement avec la vie « sédentaire » et l'environnement humain est quelque peu brutal : en effet, bien loin du paysage immense, vide d'humains et muet dans lequel le jeune berger a passé des mois et des journées paisibles où,

16. «...pour que je vous conte/comment sont au pâturage les bêtes qui transhument/ comment se couvre d'herbe les sables du Sêno/et comment, à la porte de l'été, j'ai tambouriné !/Grand-Taureau-Ceinturé-de-Blanc !/Grand-Cuivré-[rejeton] d'Hippotrague !/Jeûnons, Taureau-de-la-Cuivrée !/Par Dieu, patience !/Même en haut, pas une ondée !/la Constellation Dôri na pas encore quitté les cieux/et celle des Pleïades n'a pas encore paru/Oies d'Égypte ne sont pas encore montées et gouttes de pluie pas encore tombées/canaux ne se sont pas gonflés... » On voit ici, outre le jeu des anaphores et des épiphores, comment l'introduction progressive d'une consonne ou d'une syllabe annonce l'allitération qui va devenir dominante. 
en la seule compagnie de ses bêtes calmement occupées à paître ou à ruminer, il a pu s'adonner à loisir à ses jeux de langage si sophistiqués, c'est, au contraire, lors des grands rassemblements marquant les fêtes de transhumance que, devant l'affluence festive des villageois assemblés, il va devoir déclamer à tue-tête son poème, dans le brouhaha de la foule et du piétinement de son troupeau qui, d'un bon trot, défile dans la poussière!

Et c'est alors qu'il adopte un type de déclamation qui s'avère en accord total avec cette notion de continuum tel que texte et style le dévoilent ; ainsi, l'apprenti-poète passe brutalement du silence à la parole comme s'il laissait soudain jaillir un discours toujours latent mais laissé jusque-là tacite et qui, lorsqu'il y met tout aussi brutalement fin par un simple mi tampii, « je suis fatigué », se poursuit potentiellement tout aussi tacitement ; et certains bergers accentuent ce passage du non-dit au dit, puis sa clôture, en marquant cette délimitation artificielle du texte - lui-même dénué d'introduction comme de conclusion - par un bref coup de sifflet ; d'ailleurs, tout poème est effectivement sans fin... puisqu'il sera allongé d'année en année tant que son auteur, célibataire, pourra partir encore en transhumance ${ }^{17}$...

Outre cela, le récitant adopte une modalité de profération particulière : prenant une longue inspiration, il laisse couler jusqu'à perte du souffle, un flot rapide (effréné pour certains) de vocables parfaitement articulés, tout en usant d'une modulation de la voix pour combiner les capacités de l'émission vocale, les contraintes du mécanisme respiratoire et les structures de la langue elle-même; ce qui aboutit à une énonciation forcenée qui suit un mouvement où se superposent deux rythmes : l'un, ample, suit la respiration, l'autre, accéléré, scandé, haché, marque une intonation selon l'intensité vocale, et une accentuation dans l'articulation des mots. Toujours est-il que l'impression produite par cet exercice de virtuosité vocale et d'harmonie phonique, marqué par la neutralité d'un ambitus assez étroit et toujours égal, ne fait qu'accentuer la notion de continuum qui s'avère le point focal de cette création littéraire.

Dernier élément constitutif de ce phénomène socioculturel qu'est la profération circonstancielle de cette poésie : le fait qu'elle soit déclamée tandis que défile le troupeau, crée, pour le public qui y assiste, une incontournable impression de synesthésie entre sons et couleurs ; tout comme les mots s'enfilent en une succession sonore, chacun amenant le suivant grâce à quelques phonèmes qui lui font écho, de même les robes des bêtes enchaînent leurs combinaisons

17. Tel le poème de Moussa Golombo, composé au cours de sept transhumances successives et comportant 1150 vers. 
de couleurs et de taches, chaque vache étant suivie de son petit, dont la robe reproduit, tout en en variant légèrement, celle de sa mère. Et c'est ainsi que le cortège ininterrompu du troupeau apparaît immanquablement comme le doublet visuel de l'énonciation de ces jammooje na'i et vice versa. Et, dès lors, défilé et poème ainsi associés, prennent une dimension symbolique, celle de la représentation propitiatoire d'une fécondité assurée tant pour le bétail que pour la langue, ces deux données fondamentales, constitutives de l'identité même de ce peuple, d'après ses légendes d'origine ${ }^{18}$ : en effet, disent-elles, un frère et une sœur s'étant mis à parler une langue inconnue de leurs parents, ceux-ci, pensant avoir affaire à des djinns, les chassèrent ; dans leur errance, les enfants, ayant allumé un feu près d'un cours d'eau, virent en sortir un troupeau : ainsi devinrent-ils pasteurs ; et, isolés par leur langue, ils donnèrent naissance, par leur union incestueuse, au peuple peul.

Ainsi ces fêtes, renouvelées chaque année, apparaissent-elles, non seulement comme les temps forts d'une vie communautaire scandée par les contraintes climatiques et les nécessités économiques, mais surtout comme des rituels de perpétuation de ce qui fait la spécificité d'une communauté qui sait transmuer les contraintes naturelles conditionnant son existence en moyens d'affirmer son identité culturelle. Et c'est ainsi que, par le biais d'une manipulation particulière de la langue prise comme matériau sonore se prêtant à un art de la parole, elle transforme l'évocation de tout l'environnement qui fait son vécu habituel, et l'acte même d'énonciation de cette mise en parole, en une exhibition de la maîtrise qu'elle a acquise de ce vécu ; de plus, dans la concomitance de cette manifestation langagière avec le défilé des troupeaux - composante animale de son environnement, et élément déterminant de sa vie tant naturelle que culturelle -, cette communauté se ressoude, chaque année, à travers les productions poétiques de ses fils qui en assurent la continuité dans ses composantes essentielles : le pastoralisme et la langue. Quant aux jeunes bergers, c'est aussi à l'occasion de ces fêtes qu'ils peuvent, en exhibant leur savoir-faire pastoral et leur savoir-dire linguistique, démontrer, dans une sorte d'examen de passage public, leurs compétences tant dans le monde naturel que dans celui de la culture, et se placer comme dignes héritiers et sûrs passeurs de la tradition peule.

Tout, dans ce genre poétique, est ainsi mis en œuvre pour atteindre un au-delà des mots : ce que disent les mots n'étant que l'évidence du vécu, il convient alors de recourir à d'autres moyens pour les investir d'un pouvoir signifiant plus percutant et plus approprié aux circonstances quasi rituelles de leur énonciation,

18. Voir Seydou, 2018. 
tous ces moyens concourant à créer une perspective de perpétuation ad vitam aternam de cette société, fidèle à ses origines et à sa culture.

\section{Des jammooje na' $i$ au mergol}

Un troisième cas de figure peut illustrer le traitement écopoétique d'un même sujet selon les contextes différents impliqués par le statut du poète ; un exemple nous est fourni par le poète mergoowo Hammadi Hamma, pasteur invétéré qui, en 1973, bien que vénérable vieillard, parcourait encore la région, muni de sa boulade et vêtu, comme un jeune pâtre, du poncho de laine brune qui laisse les jambes nues, pour, soucieux de l'état de son troupeau, surveiller ses jeunes bergers, en les rattrapant de-ci de-là au cours de leur transhumance... Excellent poète, il pratiquait le mergol avec brio, sur tous sujets : de l'évocation émue des belles de sa jeunesse au récit circonstancié d'anecdotes, de querelles..., mais avec une prédilection pour les thèmes pastoraux.

Bon nombre de ses poèmes traitent du même sujet que les jammooje na'i, ce qui permet de cerner les différences de traitement stylistique introduites par les différences de circonstances dans lesquelles chaque genre est pratiqué : circonstances de création tout autant que de performance.

C'est ainsi que, dans les nombreux mergi de ce poète dédiés aux bovins et à la transhumance, même lorsque tout le vocabulaire, les images, les actions évoquées sont les mêmes que dans les jammooje na'i, on décèle immédiatement une structure narrative qui est manifestement absente dans ceux-ci ; et, au style vocatif des jammooje na' $i$, fait place ici, la syntaxe simple du récit.

C'est que le mergol a pour dessein d'informer, de transmettre... et notre poète septuagénaire, tout imprégné qu'il soit du souvenir des transhumances de sa jeunesse, les raconte dans un tout autre but que d'exhiber ses prouesses pastorales et verbales, aux fêtes de transhumance. Dans le mergol, dont la visée informative reste latente, l'événementiel joue un grand rôle ; y est décrit le déroulement du vécu du berger et de son troupeau, ponctué de l'indication précise des lieux parcourus, la description détaillée des moindres « aventures », le souvenir de rencontres aimables ou... querelleuses..., et tous ces « événements » gardent place à côté de la simple activité pastorale ${ }^{19}$; ainsi au cours d'un récit de la traversée d'un cours d'eau, le poète précise :

19. Voir annexe 3. 
Bélébélé ${ }^{20}$ avec un grand taureau qui faisait face aux vagues au point qu'éclaboussures sautaient comme étincelles sur Bélébélé filant dans l'eau, Bonyé $^{21}$, taureau qui faisait flic-flac dans le fleuve, alors qu'un taureau Ibis traversait la grande île aux lavandières... et, quand Lamâssi fit sombrer des bonnets de laine, que je vis tortillons de tissus suivre le fil de l'eau au plus profond du fleuve, le courant en entraînant certains, qui agrippaient les queues, c'est alors seulement que je vis Nouhou tout seul en tête : et lorsque les bêtes arrivaient sur la rive où accoster, Nouhou avait déjà une bonne avance sur eux jusqu'au moment où un gros mâle monta sur la berge et entreprit un trot de parade :

les gens sont restés jusqu'à ce que je reprenne pied, allant vers la rive et, comme l'eau avait atteint ma taille des cadeaux de cola m’ont trouvé, encore dans le fleuve : quatre-vingts noix de Aïssa, un paquet de cola de Hammâti, et elles dirent : «Vous avec le travail ${ }^{22}$ ! » De la cola, en tout cas, on a été rassasié !

Interviennent aussi, dans ces récits circonstanciés, des scènes dialoguées ${ }^{23}$ :

[les bêtes] ont pilonné les terres sablonneuses puis poursuivi leur route.

La butte d'Assina, toute sonore du cri des grues couronnées surplombe Adiou, le grand lac [bordé] d'acacias.

C'est là que je rencontrai Daramassa, un Bella, à qui je dis : « Arrête, Bella, que je te pose une question : je cherche un grand bras d'eau où s'abreuvent [mes] mâles. » Il dit : «Voilà là-bas des bouleaux qui surplombent l'eau !

20. Bélébé est un taureau.

21. Bonyé, comme, plus haut Diéliba et, plus bas, Lamâssi sont des noms de bateaux, attribués à des taureaux qui sont de bons nageurs.

22. Formule de salutation traditionnelle. Le don de noix de cola est traditionnel dans toutes les occasions où l'on veut honorer une personne.

23. Cf. Seydou, 1991, p. 255. 
Mais s'y trouvent aussi sagaies acérées et canons de fusils !

Détournes-en tes pas, enfant Ouroullo ${ }^{24}$ ! »

Je me raclai la gorge et crachai sur le [chien] au boubou sale :

«- Les hostilités ouvertes tout là-haut se déchaîneraient-elles,

et dussent les balles être comme abeilles fusant de toutes parts,

Le Grand-à-Tête-Noire doit brouter la brousse hirsute de Alawâwi ${ }^{25}$. »

Quant aux choix stylistiques, le mergol privilégie bien les figures habituelles de toute langue poétique comme le montrent ces quelques vers :

\section{Sayge oole oolal hurgaawe \\ ana jaayi deedi ana liba deebaaje \\ deeba hib6a Deeboy woortaali \\ nawre woodi baalol hodi baawe \\ no debbi nyaamri illa ngel e deebaaje \\ nyaaraama nyaale Ndentaaka jam nyallu! \\ Mido anndi toownde Banngu yo tolo Jalli ${ }^{26}$...}

Et, certes, comme dans les jammooje na'i, la recherche de redondances phoniques est évidente ; mais elle s'organise plus en répétitions faciles à l'intérieur d'un vers ou en rebondissements d'un vers à l'autre, qu'en la recherche obsessionnelle d'un tuilage.

En fait, les jeux phoniques ont bien, ici aussi, pour objet la musique des mots et leur rythme, mais cela, pour leur seule dimension esthétique, adjuvant aimable du discours, le mergol ayant une tout autre fonction que les jammooje na'i. Car, même lorsqu'il s'agit de l'évocation d'un environnement strictement identique, comme ici, celui de la transhumance et du pastoralisme, le propos du poète mergoowo et celui du jeune berger s'inscrit dans un vécu et des perspectives particulières à chacun ; ce qui façonne l'expression littéraire et le type de performance propre à chaque genre.

24. Groupe peul auquel appartient le poète.

25. Nom de lieu évoquant l'expression Alla waawii : «Dieu a pu ! » (i.e. Dieu est Puissant).

26. La Fauve-au-Ventre-Blanc de la Grande-Fauve-Cornes-en-Ogive/rentre du pâturage, panse couchant herbes des cuvettes/et se repaît, tout près du Dêboy, sans détour !/C'est à une mare avec herbe baalol, lieu de séjour d'oies d'Égypte,/qu'ont pâturé les femelles depuis cette petite zone dans les cuvettes/et s'est égaillé un vol d'aigrettes, à Ndentâka, bonne journée !/Je sais que la butte de Bangou est un îlot réservé aux Dialli. 
L'examen de ces deux genres poétiques est particulièrement éclairant sur, d'une part, les rapports qu'une société établit entre environnement et littérature et, d'autre part, les fonctions attribuées à la langue, selon la qualité de ces rapports.

Ainsi, on a vu que, dans le mergol, pratiqué par des poètes établis, reconnus comme tels dans leur communauté, la langue, si habilement et esthétiquement ordonnée soit-elle, conserve à ses mots, prioritairement, leur propriété et leur fonction sémantiques. Ces textes ont, une intention informative latente : ils sont prétextes à fixer et répandre une sorte de compendium du savoir concernant l'actualité de la vie de la région en même temps que tous les composants de l'environnement : naturel, topographique, historique, culturel, linguistique... Ici, l'éco-poétique se comprend comme une projection de l'environnement global, tel qu'il est vécu, dans l'expression poétique et une utilisation des ressources esthétiques et mnémotechniques de l'art poétique pour assurer à la communauté une connaissance de l'ensemble de son environnement et la circulation de son patrimoine lexical.

Par contraste, le genre des jammooje na'i, pratiqué, lui, par les jeunes bergers, poètes occasionnels, comme un exercice culturel strictement circonstanciel, dévolu uniquement à une performance publique déterminée et reproduite annuellement, a une fonction tout autre. L'environnement est, dans ce cas si évident et si continûment présent que prendre la langue comme simple moyen de « le dire » et d'en assurer la mémoire ne se justifie pas suffisamment ; reste alors à faire jouer à la langue un rôle supplémentaire en recourant aux potentialités extra/ultra-sémantiques que lui permet une exploitation esthétique de sa matière phonique ; c'est ainsi que sont choisis, parmi les tropes habituels de l'expression poétique, ceux qui s'accordent le mieux avec la signification totale et la finalité de l'évènement auquel est destinée la déclamation de ces poèmes.

On voit ici comment l'écopoétique trouve chez les Peuls sa double expression, dans ces deux genres complémentaires qui utilisent tous deux les jeux de la rhétorique poétique, mais cela en fonction de l'objectif culturel assumé par chacun. Ainsi :

- pour communiquer et perpétuer un savoir collectif propice à la cohésion de la communauté, l'un conserve à la langue sa fonction sémantique et informative, en traduisant en paroles l'environnement dans toutes ses composantes, mais cela sous une forme poétique qui assure une certaine jouissance esthétique en même temps qu'elle favorise la mémorisation ;

- pour s'inscrire dans la portée tout à la fois sémantique et pragmatique d'une cérémonie festive collective, l'autre tire parti de toutes les composantes de la matière linguistique, en particulier de sa composante acoustique, pour 
lui faire atteindre un au-delà des mots en participant à un acte qui, dans sa dimension symbolique, dépasse le simple objectif $\mathrm{du} \ll$ dire ».

Mais c'est aussi là, l'illustration d'une exploitation de la langue qui est particulièrement liée à une situation socioculturelle d'oralité. Certes, partout, l'expression poétique est éminemment liée à l'oralité - « de la musique avant toute chose ${ }^{27} \gg-$, mais choisir de rendre « signifiante », en soi, la « forme » des mots, par-delà leur valeur sémantique et leur enchaînement syntaxique, en jouant prioritairement sur leur valeur « acoustique » n'est possible qu'en situation de performance orale.

Reste enfin à comprendre les raisons d'un tel « investissement » dans la langue et ses potentialités tant sémantiques qu'esthétiques et... pragmatiques, chez cette population qui, d'ailleurs, se distingue, dans cette région de l'Afrique, par la diversité et la multiplicité des genres littéraires qu'elle pratique.

Il est, dès l'abord, exceptionnel et symptomatique qu'un peuple se donne pour origine l'apparition d'une langue nouvelle. Pour les Peuls, on peut présumer que, éleveurs de bovins voués au pastoralisme - comme l'indique d'ailleurs la légende - et originellement nomades, ils n'aient eu comme matériau toujours disponible sur lequel pouvoir exercer un art, que leur langue et sa musique ${ }^{28}$, leur mobilité obligée leur interdisant tout encombrement superflu. Et de fait, à la différence des populations sédentaires voisines, ils n'ont aucune production artistique « matérielle » : statuaire, masques...

Par ailleurs, au gré de leur sédentarisation progressive dans toute la zone sahélienne, ils se sont trouvés en contact avec des populations et des langues très diverses et seule leur langue reste, au-delà de leur dispersion, le point commun préservé, marque de leur unité.

Enfin, dans cette région du Massina où, sédentarisés, ils sont restés éleveurs et doivent pratiquer une transhumance particulièrement longue dans le temps et dans l'espace, ils se retrouvent confrontés à cette double situation qui les met en contact avec l'environnement tant urbain que sauvage. Motivés, en outre, par le souci de préserver et de pérenniser leur culture, ils ont ainsi choisi ces deux moyens poétiques pour répondre à leurs « besoins » socioculturels et, tout

\section{Cf. le poème de Verlaine : «Art poétique ».}

28. Pour ce qui est de l'art musical, outre la langue et le chant, les seuls instruments pratiqués (et autorisés aux jeunes gens tant qu'ils ne sont pas socialement intégrés, par le mariage, dans le monde des adultes) sont le pipeau en tige de mil (poopiliwal) et le petit luth monocorde (moolaandu). 
autant, artistiques, en sollicitant les différentes faces et les diverses potentialités de la langue.

\section{Annexe 1}

Extrait d'un poème mergol (de 190 vers) de Kaw Bî de Ndoukoye. Enregistré à Diondiôri (Mali) le 26/02/1973.

\section{/.../}

Mido tuumi yahan luumo

ndawa ngaddaa ndaamaari

Afel ngel nee almeeti !

Furfuru ${ }^{30}$ fuddoode luumo!

Joon kaa mi ittii dum fuusu!

Dammbi baali e danngaaji

daade e dadi naata luumo

teren kanyum e tenndeeje

temeeji ana naata luumo.

\section{/.../}

J'imagine qu'on doive se rendre au marché :

de bon matin tu te mets en route et tu amènes un taureau ndâma ${ }^{29}$.

La première petite chose, bien sûr, des allumettes !

Beignet, c'est par ça qu'on commence au marché !

Mais maintenant je cesse de plaisanter!

Cordes pour moutons et sacoches en cuir

cordes à veaux et cordelettes à entrave viennent sur un marché

terrain avec grils à viande ${ }^{31}$

tamis sont apportés sur un marché.

29. Ndaamaari : ce terme introduit une équivoque puisqu'il désigne normalement un python mais peut aussi évoquer un bovin de race ndaama, petite et sans bosse, rare dans cette région où les bovins sont tous des zébus (bos indicus); équivoque volontaire que confirme le vers 5 : « Maintenant je cesse de plaisanter !»

30. Mot bambara : furrufuru ou foroforo, « galette ou beignet de mil ou de haricot ».

31. Des vendeurs de viande grillée s’ installent sur les marchés. 


\begin{tabular}{|c|c|}
\hline Puccoy-poore ${ }^{32}$, poppooji ${ }^{33}$ & Bicyclettes, motos, \\
\hline potiije ana naata luumo & marmites sont apportées au marché \\
\hline mobil kanyum e motereeji ${ }^{34}$ & une automobile et aussi des moteurs et \\
\hline mottirde ana naata luumo. & $\begin{array}{l}\text { un panier pour fileuse }{ }^{35} \text { sont apportés } \\
\text { au marché. }\end{array}$ \\
\hline Parse kanyum e pantif ${ }^{36}$ & Parapluies et aussi pantoufles \\
\hline pade kosuuje & sandales de cuir, \\
\hline paali njuumri manaajien & gourdes de miel et objets en plastique \\
\hline marcamaaji ${ }^{37}$ kerepu'en keeda & fouets en peau de lamantin, chaussures \\
\hline banye. & à semelles crêpe y voisinent. \\
\hline $\begin{array}{l}\text { Lootorkooji lonnjorde ga'i bujji } \\
\text { ukkee e luumo }\end{array}$ & $\begin{array}{l}\text { Éponges végétales, lunettes, bœufs } \\
\text { d'embouche sont introduits sur un } \\
\text { marché. }\end{array}$ \\
\hline Baddi e na'i baalikiiji & Petits veaux et vieilles vaches \\
\hline kaabi kanyum e ga'i kayeeji & taures ainsi que taurillons \\
\hline ko mawdi biye ${ }^{38}$ koo yo bijji! & $\begin{array}{l}\text { ce qui vaut force billets, ce sont jeunes } \\
\text { génisses! }\end{array}$ \\
\hline Noon mi yi'ii toon biskit & Ainsi ai-je vu là-bas des biscuits \\
\hline
\end{tabular}

32. Textuellement « petits chevaux à pneus » (poore : caoutchouc).

33.poppooji : par onomatopée, ce mot désigne les motos.

34. mobil : français 《 automobile »; motereeji : « moteurs », désigne soit des moteurs pour pirogues soit, par synecdoque, des pirogues à moteur.

35. Panier de paille contenant tout le nécessaire pour filer, fuseaux, quenouilles.

36. Mots français : « parasol », « pantoufles ».

37. Français « crêpe », comme plus loin, butalaaji : «bouteille »et, plus loin, sigireeti : «cigarette », simsi ou simiisi : « chemise », pomaati : « pommade », lason : «la chaux », pengeleesi : « épingles », tamaati : « tomates »et, plus loin, kamsi : « camisoles », karwaasi : « cravache ».

38. biye : «billet » et, plus loin, biskit: « biscuit ». 
mi yi'ii cancorde e cagiije

aaman kanyum ${ }^{40}$ e alluuje.

Walaa ko adotoo wacco

wattanam hen kolmuuje

ngaddanaa kam kojolooje.

Fay konkeeje ngon luumo

diisi e diwle e dimaadi.

Ndirkitaa too e njaabaandi

njaaraa gaa'en yo cekke

ceemunje e boggi ceelaadi

boobo-juruuji e boliiji j'ai vu des peignes et des paniers ${ }^{39}$

de l'eau avec aussi des tablettes ${ }^{41}$.

Rien qui soit plus primeur que le souchet ${ }^{42}$

ajoutes-y pour moi des fruits d'ébénier du Sénégal ${ }^{43}$

apporte-moi des feuilles de bouleau d'Afrique ${ }^{44}$.

Il y a même des fruits de konkeehi ${ }^{45}$ sur un marché

des cœurs de palmiers ${ }^{46}$ et des charges et des chevaux de race.

Pousse un peu par là, et c'est de la pâte de jujubes ${ }^{47}$,

avance par ici : ce sont paillassons

harpons et cordes en lanières de cuir

cordes en écorce de baobab et gourdes en calebasses

39. Hauts paniers à fond carré, rigide, et bord supérieur circulaire.

40. aaman: « eau » en tamasheq.

41. Tablettes à écrire, en bois, utilisées par les écoliers d'école coranique.

42. Petits tubercules comestibles de diverses plantes poussant au bord de l'eau.

43. Diospyros mespiliformis Hochst (Ebanaceae) ; les fruits de cet arbre sont comestibles et se vendent ; ceux qui, non cueillis, tombent de l'arbre, sont secs, noirs et très sucrés.

44. Anogeissus leiocarpus (DC) Guill. et Perr. (Combretaceae) ; les cendres sont utilisées pour le tannage et les feuilles pour la teinture marron dite wolo.

45. Detarium senegalense J.F. Gmel. ou microcarpum Guill. et Perr. ; les fruits comestibles sont vendus.

46. Les jeunes pousses de rôniers sont comestibles : on plante les noyaux, on les fait germer et on en consomme les germes.

47. Les jujubes secs sont pilés et donnent une farine dont on fait des sortes de galettes. 

bulki kanyum e butalaaji ${ }^{6}$
cruches et aussi bouteilles
uurdi kanyum e uureteede
encens et aussi gommes aromatiques
dacce kanyum e siraa-dakka
gomme arabique et aussi tabac pilé
sigireeti tan kanyum e simsi
cigarettes mais aussi chemises
/.../
/.../

\section{Commentaire}

On remarque que, à la différence des poèmes pastoraux, les allitérations (en parallélismes, rimes, répétitions...) se succèdent ici, sans la recherche d'un tuilage, l'intention de ce genre étant diffférente : il s'agit en effet tout à la fois de transmettre une connaissance des « choses et des mots » et, ce faisant, faire étalage tant de l'étendue de son savoir que de son talent personnel à manipuler la langue dans toutes ses potentialités formelles.

\section{Annexe 2}

Extrait d'un poème jammoore na'i (de plus de mille vers) de Moussa Gouloumbo (inédit).

Enregistré à Oumméré (Mali) en 1973.

\section{/.../}

Cade! Karbotoodi buurtol

kaptotoodi buukal

senngo bunal caryal

di ngoni di kulaali ndoggaali ndagaali!

Ndalaaji ndadaali hakkunde Kogoni e kolaade Addeyna!

Paate humoo
Salut ! Bêtes qui se lancent en masse sur la piste,

détalent dans bourbier,

et, aux côtés de

Gros-Gris-Tourdille-Haut-Encorné,

sont bêtes sans peur qui n'ont ni fui ni stationné !

Moutons noirs ne les ont point devancées entre Kogoni et plaines d'Addeyna !

Pâté se ceint les reins 
palaadi kooloo so mi jirgitii kolon:

illa tobaali tudaali gidaali ngirgirtaali gine ngiwjaali

kunyoo ngada guurtol kama wula kolla Maasina kaawde :

no kogolal maajori tiwre

mantotoongal se tiidii

se tiwre ndee haadii

haala welii!

Na'i paloo e buurtol

pardinee murta

ndokkee palaasi Murja nde

kolaade Mulay'eeli

girrawal

nguli yaarii

duule yaafi

dulbe puddii

duluuji onngii

dumari koorii

koyaaba oonii, kogoli oymii e tiwre...

gayta, naange oolal caryal!

En taw came nduumaali

Ndukaala aawaali et les Marquées-de-Croix sont confiantes, dès que je ravive l'ardeur des troupes : alors qu'il n'a point encore plu, qu'eau n'est pas apparue, que tonnerre n'a pas retenti de-ci de-là, que cigognes-marabouts n'ont pas pataugé,

elles se mettent en route, prenant draille de front dans la chaleur, et font au Massina voir merveilles :

comment Gros-aux-Yeux-d'Albinos a défilé au pas sur l'étape

lui qui se vante, alors que l'heure était dure et rude la route mais en parler, bien agréable!

Vaches barrent la draille on les fait parader, elles se rebellent on leur fait place à Mourdia dans les plaines de Moulaye-Êli, et le grand Guirradio a ruisselé de sueur nuées se sont gonflées hivernage s'est annoncé tourbillons de poussière ont divergé Ibis-Sacrés ont jeûné gros taureaux ont mugi, Cornes-ArquéesPointes-en-Proue sont harassés sur l'étape... gémis plaintivement, soleil de Grand-Fauve-aux-Cornes-en-Lyre ! Nous avons trouvé que tiami ${ }^{48}$ n'avaient point encore reverdi que Ndoukâla n'avait pas encore semé

48. Pterocarpus lucens Lepr. ex Guill. et Perr. (Papilionaceae) ; arbuste dont le feuillage fournit un bon fourrage. 
ndunngu jannjaali

Jalli kuncaali

jabbe uddaali! qu'hivernage ne battait pas encore son plein

que les Diall n'étaient pas encore en route et les poquets pas encore refermés!

Exemple d'enchaînements sonores

\begin{tabular}{|c|c|}
\hline $\begin{array}{l}\text { 1. Aan jey denngorde Deeba } \\
\text { 2. aan uddata depporde mayru } \\
\text { 3. gila Nyolngol faa e Seeri. } \\
\text { 4. Do muuy-daa fuu tuppu seko, } \\
\text { 5. Se66e mbaawaa lunndaade! } \\
\text { 6. Gila bama Seeka Jafaraa6e } \\
\text { 7. faa yottoo Seeka calde } \\
\text { 8. camdo ma hiraay e Se66e ! } \\
\text { 9. Jaari uddata jaanaaje } \\
\text { 10. Jaari tuufata jammbuuje } \\
\text { 11. e jamde juwirde konndooji } \\
\text { 12. e kocokoco piildaado konngi } \\
\text { 13. e kobaaje awrude pinyaaje } \\
\text { 14. kanyum e koosiije liddi... }\end{array}$ & 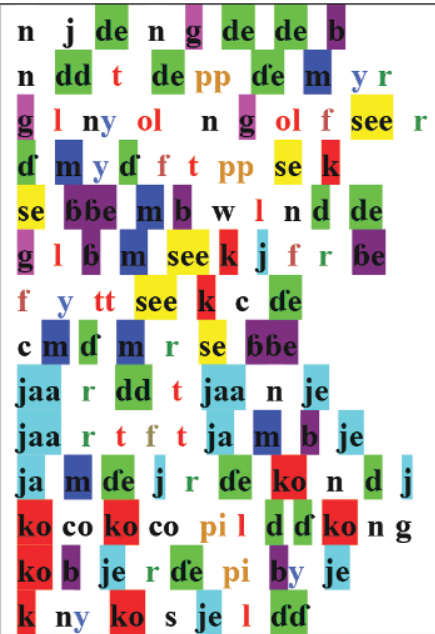 \\
\hline
\end{tabular}

On voit comment, grâce aux $\mathbf{g}$ et $\mathbf{n}$ persistant de 1 à 3 , on passe

d'une dominante de/de à une dominante $\mathrm{l}$, ol (3), puis grâce aux $\mathbf{d}, \mathbf{y}$ et $\mathbf{f}$ (en 3 et 4 ), à une dominante se/see, $\mathbf{m}$ et $\mathbf{b}$ (de 4 à 8 ), et grâce aux f, $\mathbf{d}$, $\mathbf{r}$ et $\mathbf{t}$ persistants de 6 à 9 , à la dominante $\mathbf{m}$ et $\mathbf{j a} / \mathbf{j e}$, et enfin, grâce à la persistance de $\mathbf{j}, \mathbf{m}, \mathbf{d e}, \mathbf{r}$ et $\mathbf{n}$, à la dominante en $\mathbf{k o} .$.

\section{Annexe 3}

Extraits d'un poème de 576 vers, du mergoowo Hammadi Hamma Bari, éleveur septuagénaire, enregistré à Sindégué, en mars 1973.

\section{I.../}

Nde bijji kaabunoo biilal Seewa

mi seri wooto Belebele huri wooto 
gordi mawdi njeryidi masinaaji so di immodiima faa kare ana iida hono kanuuru immii dow kaaye. Ngel Gaabdo huyri bujji ngada ganngiije gaanyaaji tummba ngel Tufi nyallirta. Banal e nyaale mbatti kam di mbii Nyaaba jam nyallu leydi mawngal nyaaruuji! Di kammbi weendu mido wedoo kaabi Edi ceyi e seeno edi mbobsoo seewndu seebuure beeli seyoseyo sera Beeyna. Serinda jawle yim Senndera Jallo!

Di boowdi perre biidi e pete gawde garci Baaba Haawa e Hammaati fay so moomtu-don debe jinootoodo faa mi yeewa baawdo di yeyinde debe e sappo fun sappii yo yinotoobe yoga saawaama illa yappere Saasi yoga soyyitiima illa ndi soobaaki nde Jeliba sirtunoo be cikkunoo siido Jara sikku dee ko wonnoo koo woodii nde arannde ndee mo wada arkummbeeje illa teentilaaka tawi Jara tebbaama Belebele e ganndotoongal bempeeje faa picce nyaara dow Belebele nyaarta Bonye gadoori bojobaja ley maayo faa dumari tacci duungal loonnoobe nde Lamaasi yurbini laafaaji njii-mi tekke njokki e tewtiwri teere wubba yoga teb6oo laace kanaa njii-mi Nubu teeli e hoore se di ngarata faandude nde tawi Nubu faddaama faa goral Yeeji dartini fankiiru be keddii faa so ndartinu-mi e danyeere so ndiyam yottinoo kam taadorde faa caadaaje tawi kam e ley maayo hemre Aysa fufu goro Hammaati be mbii «onon e golle! » Goro kaa haaraama! 


\section{Traduction :}

Une fois génisses lassées du grand campement abandonné de Sêwa je donnai un seul coup de sifflet, Bélébélé ${ }^{49}$ poussa un seul grognement et grands mâles ont d'un seul élan lancé leurs machines tous ensemble mis en branle au point que vibre le sol dur comme un coup de canon sur les collines.

Le chéri de Gâbdo ${ }^{50}$ est enthousiaste car gros bœufs sont comme piliers centraux de cases,

gros taureaux l'entourent, lui à qui Toufi pense tout le jour ! Gros-Noir et Aigrette ${ }^{51}$ me font compagnie et disent « à Gnâba! » et que soit bonne la journée au pays aux vastes plaines herbeuses ! Les bêtes longent un lac tandis que je navigue parmi les génisses ; elles sont allées s'incruster dans le Sêno, à siroter un air léger, herbe des lacs, buissons aux abords du Bêyna.

Mets-toi à l'écart des troupeaux et chante Sendéra Diallo !

$$
\text { /.../ }
$$

Accoutumés aux terres dures, aux ruisseaux et flaches à acacias sont les troupeaux transhumants de Bâba-Hâwa et de Hammati. Alors même que vous eûtes rassemblé quarante nageurs ${ }^{52}$ pour que je voie

celui qui pourrait le mieux les faire remonter sur la berge et que toute une cinquantaine se targuèrent d'être de bons nageurs, les uns se sont trouvés noyés dans la masse dès la brèche de Sâssi, les autres ont fait demi-tour sans qu'un meneur s'y soit même appliqué

\section{Nom d'un taureau.}

50. Il s'agit du poète lui-même ; on trouve tout au long du poème des allusions aux jeunes femmes courtisées : Gabdo, Toufi, Sendéré.

51. Les bêtes portent le nom d'oiseaux dont le plumage a même couleur et même taches que leur robe. Aigrette est ici une vache blanche comme, plus loin, Ibis a, comme l'Ibis sacré, poitrail blanc, tête et croupe noires.

52. La traversée des cours d'eau par les troupeaux fait partie des temps forts de la transhumance. La plus importante, à Diafarâbé, fait partie des événements festifs traditionnels du Mali. 
et, lorsque Diéliba ${ }^{53}$ eut filé, fendant l'eau, et qu'ils prirent ça à la légère,

Diara, certes, pensa que ce qui s'était passé était bien :

au début, il ne nageait qu'avec les jambes

mais avant qu'il se soit trouvé bien en action, Diara a été rattrapé ;

Bélébélé avec un grand taureau qui faisait face aux vagues

au point qu'éclaboussures sautaient comme étincelles sur Bélébélé filant dans l'eau

Bonyé $^{54}$, taureau qui fait flic-flac dans le fleuve

au point qu'un taureau Ibis traversa la grande île aux lavandières et quand Lamâssi fit sombrer des bonnets de laine,

que je vis tortillons de tissus suivre le fil de l'eau au plus profond du fleuve,

le courant en entraînant certains, qui agrippaient les queues, c'est alors seulement que je vis Nouhou tout seul en tête :

et lorsque les bêtes arrivaient sur la rive où accoster, Nouhou avait déjà une bonne avance sur eux

jusqu'au moment où un gros mâle monta sur la berge et entreprit un trot de parade :

les gens sont restés jusqu'à ce que je reprenne pied, allant vers la rive

et, comme l'eau avait atteint ma taille

des cadeaux de cola m'ont trouvé, encore dans le fleuve :

quatre-vingts noix de Aïssa, un paquet de cola de Hammâti, elles dirent : «Vous avec le travail ${ }^{55}$ ! » De la cola, en tout cas, on a été rassasié !

\section{Références bibliographiques}

BÂ Âmadou Hampâté \& DAget Jacques, 1984, L’Empire peul du Macina (18181853), Les Nouvelles Éditions Africaines, Abidjan, 306 p.

53. Nom d'un taureau bon nageur, donné en souvenir d'un ancien bateau ainsi nommé.

54. Bonyé, comme, plus haut Diéliba et, plus bas, Lamâssi sont des noms de bateaux, attribués à des taureaux qui sont bons nageurs.

55. Formule de salutation traditionnelle. Le don de noix de cola est traditionnel dans toutes les occasions où l'on veut honorer une personne. 
Gallais Jean, 1967, Le Delta intérieur du Niger. Étude de géographie régionale, 2 t., Mémoires de l'IFAN, nº 78, Dakar, 621 p.

Gallais Jean, 1984, Hommes du Sahel, Flammarion (Coll. « Géographes »), Paris, 296 p.

Sanankoua Bintou, 1990, Un empire peul au XIX siècle. La diina du Maasina, Karthala-ACCT, Paris, 174 p.

Seydou Christiane, 1989, « De l'art d'accommoder les mots : lexiques bilingues en forme de poèmes » in URA 1024 (éd.), Graines de parole, puissance du verbe et traditions orales, Écrits pour Geneviève Calame-Griaule, Éditions du CNRS, Paris, p. 299-328.

Seydou Christiane (présentée et traduite par), 1991, Bergers des mots, poésie peule du Massina, bilingue, Les Belles Lettres (coll. Classiques africains 24; distribué par Karthala), Paris, 357 p.

Seydou Christiane, 2013, «Espace héroïque : devises de villes dans l'épopée peule du Mali », in KeÏTA Abdoulaye (dir.), Au Carrefour des littératures Afrique-Europe, Hommage à Lilyan Kesteloot, IFAN-Karthala, p. 71-89.

Seydou Christiane, 2018, «Langue et identité. Légendes d'origine des Peuls » in Cabiers de littérature orale, $\mathrm{n}^{\circ} 83, \mathrm{p} .79-99$.

Résumé : Chez les Peuls du Massina (Mali), la situation écologique trouve, dans deux genres poétiques, une illustration des rapports entre l'environnement et cette expression langagière à vocation esthétique et culturelle qu'est la littérature. L'un, le mergol, qui traite de tout sujet, recourt à l'expression poétique - qui favorise la mémorisation - pour décrire la réalité écologique et l'impact de l'humain sur l'organisation de l'espace, dans une perspective directement informative, pour la transmission et la diffusion du patrimoine culturel et linguistique. L'autre, les jammooje na'i - louanges aux bovins -, déclamé par les jeunes bergers aux fêtes de transhumance, est directement lié à l'inscription de la vie humaine et animale dans l'espace et la réalité écologique, et s'en trouve naturellement conditionné, certes dans son contenu, mais plus encore dans sa forme, ses instances d'élaboration et de performance et enfin sa fonction ; ici, l'utilisation de la langue dans sa dimension plus formelle que sémantique investit ces poèmes d'un au-delà des mots : défilé des troupeaux et enchaînement des 
mots étant symbole de perpétuation de ce qui fait l'identité peule. L'impact de la relation à l'environnement dans l'expression littéraire s'explique sans doute par la situation originellement nomade de cette population qui, d'après ses légendes, se donne pour origine l'apparition d'une langue nouvelle et d'un troupeau de bovins ; et ces deux genres poétiques illustrent deux types de relations possibles entre situation écologique et création littéraire.

Mots-clefs : Mali, Peuls, environnement, genres poétiques, oralité, création littéraire.

Abstract: For the Massina Fulani (Mali), the ecological situation finds, in two poetical genres, an illustration of the connections between environment and this linguistic expression with an aesthetic and cultural purpose which is literature. One, mergol, dealing with any subject, resorts to the poetical expression-which favours memorization - in order to depict the ecological reality and the impact of the human factor on the organisation of space, in a purely informative perspective, for the transmission and diffusion of the cultural and linguistic heritage. The other one, the jammooje na' $\mathrm{i}$-praises to the cattle-declaimed by the young shepherds during the transhumance festivals, is directly related to the place and the role of buman and animal life in space and in the ecological reality, and turns out to be naturally conditioned in its contents of course, but even more in its form, its instances of elaboration and performance and finally in its function; here, the use of language in its formal rather than semantic dimension confers to these poems a scope beyond words: procession of herds and sequence of words symbolizing the perpetuation of what makes the Fulani identity. The impact of the connection to the environment on the literary expression can no doubt be explained by the originally nomadic situation of these people who, according to their legends, attribute their origins to the appearance of e new language and a herd of cattle; and these two poetical genres illustrate two types of possible relationships between ecological situation and literary creation.

Keywords: Mali, Fulani, environment, poetical genres, orality, litterary creation.

\section{Notes sur l'auteur}

Christiane Seydou, agrégée de grammaire, diplômée de l'Inalco (langues peule et haoussa, thèse de $3^{\mathrm{e}}$ cycle en littérature peule), directeur de recherche honoraire au CNRS (ERA246, Langage et culture en Afrique de l'Ouest, puis Llacan) a consacré ses recherches à la langue et la littérature peules du Massina (Mali) dans la Boucle du Niger. Outre son intérêt pour la lexicologie - Dictionnaire pluridialectal des racines verbales et Dictionnaire du fulfulde du Massina (parution 
CAHIERS DE LITTÉRATURE ORALE

174 Éc(h)opoétiques - $\mathrm{n}^{\circ} 87$

prochaine) -, elle a surtout voulu illustrer les différents genres littéraires pratiqués (contes, épopées, poésie religieuse et profane, poésie pastorale) en en publiant les textes les plus représentatifs, prioritairement en édition bilingue (une quinzaine d'ouvrages) et des présentations et analyses (une soixantaine d'articles). 